\title{
Scintigraphy false positive results for cardiac amyloidosis in a patient with Danon disease
}

\author{
Dmitry Antukh ${ }^{1}$, Dmitry Shchekochikhin ${ }^{1}$, Teona Rosina ${ }^{2}$, Elena Mershina ${ }^{3}$, Olga \\ Larina $^{3}$, Sergey Pasha ${ }^{2}$, Elena Zaklyazminskaya ${ }^{2}$, Valentin Sinitsyn $^{3}$, and Sergey Moiseev ${ }^{2}$ \\ ${ }^{1}$ Pirogov Russian National Research Medical University Medical Faculty \\ ${ }^{2}$ Sechenov University \\ ${ }^{3}$ Lomonosov Moscow State University
}

July 3, 2020

\begin{abstract}
In up to $10 \%$ of patients with hypertrophic cardiomyopathy, the disease is caused by genetic disorders including metabolic diseases. Sometimes clinical workup may be confusing. To our knowledge, this is the first report of false positive results of scintigraphy indicating cardiac amyloidosis in a patient with Danon disease.
\end{abstract}

Key Clinical Message

Common diagnostic approach in patients with suspected cardiac amyloidosis includes cardiac magnetic resonance imaging and scintigraphy. We report the first clinical case of false positive results of scintigraphy in a patient with Danon disease.

Introduction

We report a clinical case of a patient who had a hypertrophic cardiomyopathy of unknown etiology. We used variety of tests among which were cardiac magnetic resonance imaging (CMR) and scintigraphy. CMR showed dubious results but scintigraphy was false positive. As we know, there were no reports of scintigraphy false positive results indicating cardiac amyloidosis.

Case report

A 23-year-old man presented with leg edema, ascites, shortness of breath, history of intermittent palpitations and syncope, mild proximal muscle weakness and cognitive deficit. The patient reported that his mother died suddenly at the age of 22 years. Heart failure symptoms progressively worsened from the age of 16 years. He was earlier diagnosed with asymmetric non-obstructive hypertrophic cardiomyopathy, WPW-syndrome, and complete left bundle-branch block. He used metoprolol $25 \mathrm{mg}$ a day and irregularly torasemide without medical monitoring.

Laboratory tests revealed increased levels of creatine kinase, lactate dehydrogenase, aspartate aminotransferase, alanine aminotransferase which was believed to be caused by myopathy. Moreover, electromyography of the deltoid muscle showed the typical myopathic pattern.

CMR showed left and right ventricle hypertrophy (interventricular septum thickness of $24 \mathrm{~mm}$ ), interventricular asynchrony, and dilation of the pulmonary arteries and the inferior vena cava. Late gadolinium enhancement (LGE) imaging showed early subendocardial LGE throughout all LV segments which is not typical for HCM and amyloidosis. 
Considering patient's young age, family history, and the absence of kidney involvement and polyneuropathy, the most probable diagnosis seemed to be a glycogen storage disease. ATTR amyloidosis was included in differential diagnosis.

Further we performed scintigraphy and rectal mucosal biopsy. Bone tracer cardiac scintigraphy using 99mTclabeled pyrophosphate showed cardiac uptake consistent with ATTR amyloidosis. However, amyloid deposits were not found in biopsy specimens. Heart muscle biopsy was not performed due to high perioperative risks.

The definitive diagnosis was established after genetic tests. We found a frameshifting mutation in LAPM2 gene which means that patient had Danon disease.

Unfortunately, there is no specific treatment available for this disorder. The patient received implantable cardioverter defibrillator considering the high risk of sudden cardiac death. Symptomatic treatment included furosemide and spironolactone.

\section{Discussion}

Genetic disorders including metabolic diseases are reported to account for up to $10 \%$ of all causes of hypertrophic cardiomyopathy ${ }^{1}$. It is easy to suspect one in cases like ours however definitive diagnosis may be difficult to establish.

Our patient had features of metabolic disease: young age, family history, proximal muscle involvement, and cognitive deficit. Interestingly, our patient had WPW-syndrome - another common feature of a storage disease ${ }^{1}$. Particularly, the whole picture was rather typical for a Danon disease. Danon disease is lysosomeassociated membrane protein 2 (LAMP2) deficiency characterized by severe cardiomyopathy, mild skeletal myopathy, ophthalmic abnormalities, and variable intellectual disability ${ }^{2}$.

Interesting part of our case is that after dubious results of contrast enhance MRI, cardiac scintigraphy showed false positive results indicating cardiac amyloidosis. It is important to note that these modalities are a part of a common diagnostic algorithm ${ }^{3}$. To our knowledge, there were no reports of a case like ours. These modalities are believed to be highly specific but one should remember of rare conditions which could mimic cardiac amyloidosis.

\section{Author Contribution}

DA: wrote and revised manuscript. DS: wrote and revised manuscript. TR: revised manuscript. EM: revised manuscript and figure acquisition. OL revised manuscript and figure acquisition. SP: revised manuscript. EZ: revised manuscript. VS: revised manuscript. SM: revised manuscript.

Conflict of interest

None declared.

References:

1. Task A, Elliott PM, Uk C, et al. 2014 ESC Guidelines on diagnosis and management of hypertrophic cardiomyopathy The Task Force for the Diagnosis and Management of Hypertrophic Cardiomyopathy of the European Society of Cardiology ( ESC ). 2014:2733-2779. doi:10.1093/eurheartj/ehu284

2. Arad M, Maron BJ, Gorham JM, et al. Glycogen storage diseases presenting as hypertrophic cardiomyopathy. $N$ Engl J Med . 2005;352(4):362-372. doi:10.1056/NEJMoa033349

3. Dorbala S, Ando Y, Bokhari S, et al. EXPERT CONSENSUS RECOMMENDATIONS. J Nucl Cardiol . 2019. doi:10.1007/s12350-019-01761-5

Figure 1. CMR. 4 chamber view reveals myocardial hypertrophy up to $24 \mathrm{~mm}$ mostly in the interventricular segments (square), right side hydrothorax (asterisk), mild pericardial effusion (two asterisks).

Figure 2. Post-contrast images demonstrate subendocardial LGE in all LV segments. This pattern of LGE is not typical for HCM and amyloidosis. 

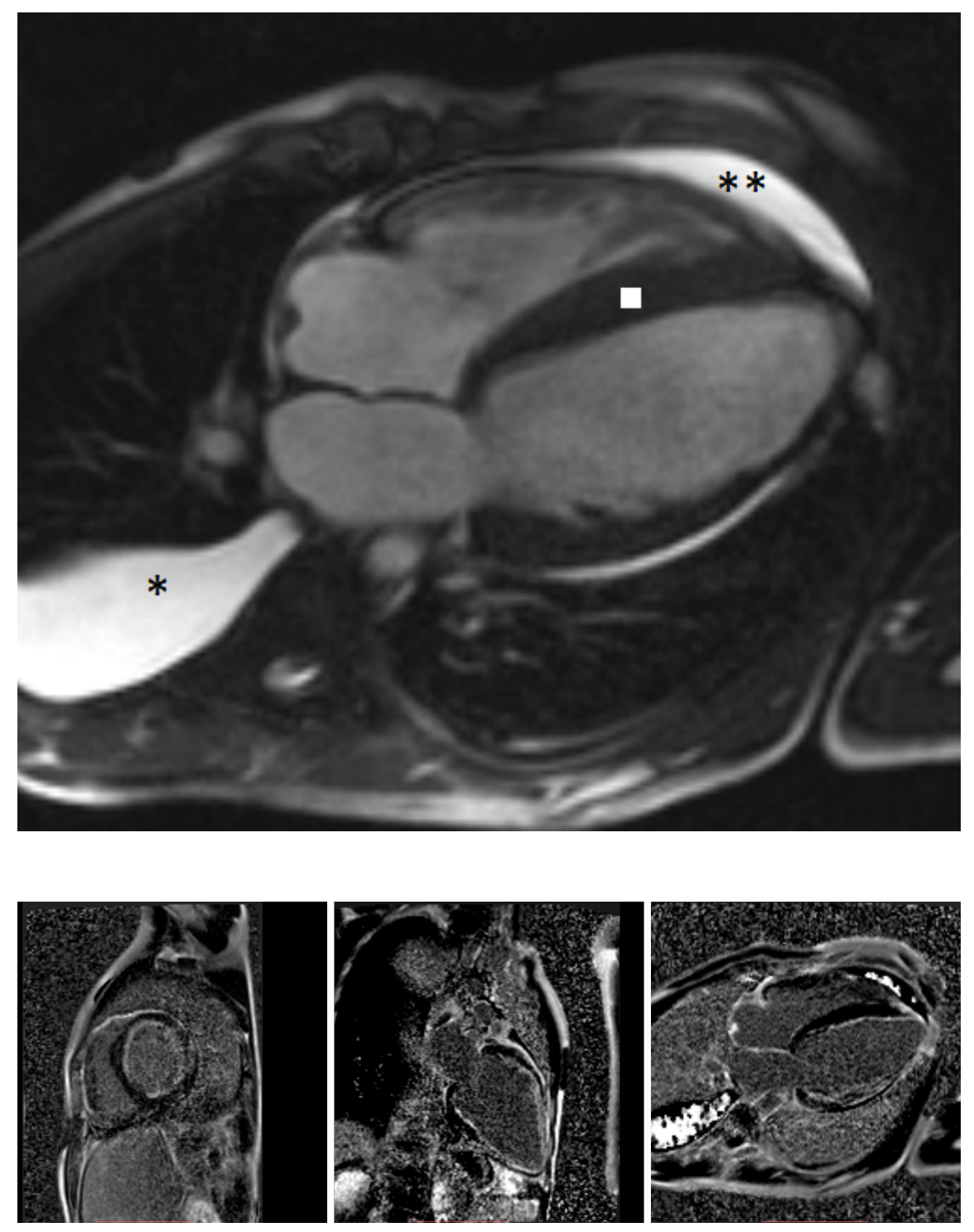